\title{
Quantum transport including nonparabolicity and phonon scattering Application to silicon nanowires
}

\author{
Journal Article \\ Author(s): \\ Esposito, Aniello; Frey, Martin; Schenk, Andreas \\ Publication date: \\ 2009-10 \\ Permanent link: \\ https://doi.org/10.3929/ethz-b-000016413
}

Rights / license:

In Copyright - Non-Commercial Use Permitted

Originally published in:

Journal of Computational Electronics 8(3-4), https://doi.org/10.1007/s10825-009-0276-0 


\title{
Quantum transport including nonparabolicity and phonon scattering: application to silicon nanowires
}

\author{
Aniello Esposito • Martin Frey • Andreas Schenk
}

Published online: 21 July 2009

(C) Springer Science+Business Media LLC 2009

\begin{abstract}
In this work, the particular and combined influence of nonparabolicity and phonon scattering on the device characteristics of a triple-gate silicon nanowire is investigated. In addition, different approximations of the retarded self-energy for electron-phonon scattering are analyzed in terms of the electrostatics, current and computational cost.
\end{abstract}

Keywords Nonparabolicity · NEGF · Scattering ·

Nanowire

\section{Introduction}

In order to give an accurate description of electronic transport in nanoscale devices, the charge carriers should be treated on the level of quantum mechanics. Simulation approaches aiming to predict the behavior of CMOS devices, usually consist of an iterative solution scheme, where the Schrödinger and the Poisson equation are solved in a selfconsistent way. In their simplest form [16, 19, 22], the Schrödinger problem is formulated in the effective mass approximation (EMA) [14], which is intended for problems whose external perturbations are smooth compared to the lattice constant of the considered material. Thus, for devices involving confinements down to the nanometer length scale, the EMA becomes questionable. A remedy is provided by advanced band structure models [15, 17, 18], as for example the $s p 3 d 5 s^{*}$ tight binding (TB) method, but their higher precision goes at the expense of the simulation time. In this

A. Esposito $(\varangle) \cdot$ M. Frey · A. Schenk

Integrated Systems Laboratory, ETH Zurich, Gloriastrasse 35, 8092 Zurich, Switzerland

e-mail: esposito@iis.ee.ethz.ch work a nonparabolicity model (NP) is discussed, which has the same computational advantages as the EMA, but incorporates a large part of the band structure effects as far as the currents are concerned. The parameters for NP are obtained from a comparison with data from a TB band structure [2]. Details are given in Sect. 2.4.

The second part of this work covers the influence of phonon scattering (SC) on the device characteristics. After a brief outline of the non-equilibrium Green's functions formalism (NEGF) [20, 21] in Sect. 2.5, which allows the introduction of scattering mechanisms on a perturbative approach, the results of different approximations for the retarded self-energy compared to the full problem are discussed in Sect. 3.3.

Finally both NP and SC are combined (NPSC) in a simulation of triple-gate silicon nanowires to study the joint impact of band structure and scattering effects on the current. The detailed discussion of NPSC, which is the main focus of this work, can be found in Sect. 3.4.

\section{Theory}

\subsection{Decoupling of the Schrödinger equation}

The kind of devices which can be treated within the transport model used in this work mainly consist of a given transverse shape which is uniformly continued along the transport direction as shown in Fig. 1(a). In the case of onedimensional electron gases, i.e. quantum wires, the lateral confinement is accounted for by Dirichlet boundary conditions while open boundary conditions are applied at the front and the end of the device in order to enable transport. The resulting Schrödinger problem needs not to be treated as 


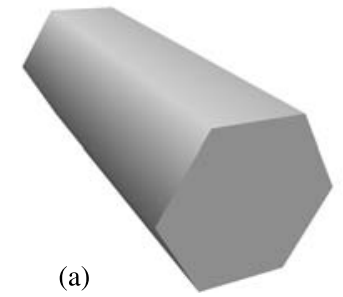

(b) $\mathbb{R}_{x}$

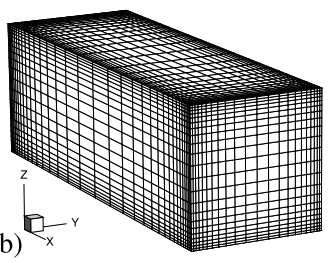

Fig. 1 (a) Schematic representation of a typical device geometry which can be treated within the transport model used in this work. The prismatic shape represents a key ingredient. (b) Tensorial grid discretization used in this work for quantum wires having a square cross section

an eigenvalue problem as the Hamiltonian of an open quantum system possesses a continuous spectrum. The equation can be solved as a boundary value problem for an appropriate energy which is known to reside in the spectrum of the Hamiltonian. Assuming that the channel region consists of silicon grown along the $\langle 100\rangle$ direction the EMA envelope equation [14] reads

$$
\underbrace{\left[-\frac{\hbar^{2}}{2 m_{e}} \nabla^{\mathrm{T}} \mathbf{M} \nabla+U(\mathbf{r})\right]} \Psi(\mathbf{r})=E \Psi(\mathbf{r}),
$$

\section{$H(\mathbf{r})$}

where $U(\mathbf{r})$ contains both the electrostatic potential and the conduction band edge, and $\left\{1 / m_{x}, 1 / m_{y}, 1 / m_{z}\right\}$ denote the diagonal entries of the inverse effective mass tensor $\mathbf{M}$. In the case of silicon the effective masses $\left\{m_{x}, m_{y}, m_{z}\right\}$ can take the values 0.19 or 0.91 depending on the considered conduction band valley. The boundary conditions with the transport direction along the $x$-axis are given by

$\left.\Psi\right|_{x=0}=f_{1}(y, z)$,

$\left.\frac{\partial}{\partial x} \Psi\right|_{x=0}=f_{2}(y, z)$,

$\left.\Psi\right|_{\mathbf{r} \in \Gamma}=0$,

where $\Gamma$ denotes the surface along the $x$-axis and $f_{1}$ and $f_{2}$ are complex valued functions defined on the $y z$-plane. The solution of the problem stated in (1), (2), (3), and (4) requires a subdivision of the transport direction into slices according to

$$
\begin{aligned}
& \mathbf{I}(x, n, N) \\
& \quad \equiv \begin{cases}{\left[x_{0},\left(x_{0}+x_{1}\right) / 2\right],} & n=0, \\
{\left[\left(x_{N-1}+x_{N}\right) / 2, x_{N}\right],} & n=N, \\
{\left[\left(x_{n-1}+x_{n}\right) / 2,\left(x_{n}+x_{n+1}\right) / 2\right],} & \text { otherwise }\end{cases}
\end{aligned}
$$

for a given set of points $\left\{x_{n} \mid 0=x_{0}<x_{1}<\cdots<x_{N-1}<\right.$ $\left.x_{N}=L_{x}\right\}$. The potential $U(\mathbf{r})$ from (1) is approximated by a set of piecewise constant functions $U_{n}(y, z) \equiv U\left(x_{n}, y, z\right)$ defined on the corresponding slice $\mathbf{I}(x, n, N)$ yielding a simplified Schrödinger problem of the form

$$
\left(-\frac{\hbar^{2}}{2 m_{e} m_{x}} \frac{\partial^{2}}{\partial x^{2}}+\widehat{\mathbf{H}}_{n}\right) \Psi(\mathbf{r})=E \Psi(\mathbf{r})
$$

where

$\widehat{\mathbf{H}}_{n} \equiv\left[-\frac{\hbar^{2}}{2}\left(\frac{\partial}{\partial y} \frac{1}{m_{y}} \frac{\partial}{\partial y}+\frac{\partial}{\partial z} \frac{1}{m_{z}} \frac{\partial}{\partial z}\right)+U_{n}(y, z)\right]$.

After using the ansatz

$$
\begin{aligned}
\left.\Psi(\mathbf{r})\right|_{x \in \mathbf{I}(x, n, N)}= & \sum_{i}\left(a_{i}^{n} e^{i k_{i}^{n}\left(x-x_{n}\right)}\right. \\
& \left.+b_{i}^{n} e^{-i k_{i}^{n}\left(x-x_{n}\right)}\right) \Phi_{i}^{n}(y, z)
\end{aligned}
$$

the problem given in $(6)$ reduces to

$$
\widehat{\mathbf{H}}_{n} \Phi_{i}^{n}(y, z)=\underbrace{\left(E-\frac{\left(\hbar k_{i}^{n}\right)^{2}}{2 m_{x}}\right)}_{\epsilon_{i}^{n}} \Phi_{i}^{n}(y, z)
$$

and

$k_{i}^{n}= \begin{cases}\frac{\sqrt{2 m_{x}\left(E-\epsilon_{i}^{n}\right)}}{\hbar}, & E-\epsilon_{i}^{n} \geq 0, \\ i \frac{\sqrt{2 m_{x}\left(\epsilon_{i}^{n}-E\right)}}{\hbar}, & \text { otherwise. }\end{cases}$

After solving the transverse problems given in (9) the total wave function $\Psi(\mathbf{r})$ to a given energy $E$ can be written as

$$
\begin{aligned}
\Psi(\mathbf{r})= & \sum_{n=0}^{N} \chi_{n}(x)\left(\sum _ { i } \left(a_{i}^{n} e^{i k_{i}^{n}\left(x-x_{n}\right)}\right.\right. \\
& \left.\left.+b_{i}^{n} e^{-i k_{i}^{n}\left(x-x_{n}\right)}\right) \Phi_{i}^{n}(y, z)\right),
\end{aligned}
$$

where $\chi_{n}(x)$ is the characteristic function on $\mathbf{I}(x, n, N)$. The remaining task consists of determining the coefficients $\left\{a_{i}^{n}, b_{i}^{n}\right\}$ from (10) as well as finding a suitable implementation for the solution of the transverse problems described in (9).

\subsection{Solution of the transverse problem}

Several approaches are available in order to solve the Schrödinger problem given in (9). Especially in the case of arbitrarily curved transverse shapes as shown in Fig. 1(a) the finite element method (FEM) [13] turned out to be a useful tool. However, as in this work the attention is focused to devices with a square cross section a finite difference (FD) scheme is preferable as it is a cheaper alternative to the FEM from an implementation and performance 
point of view. For this purpose the transverse domain can be meshed with a tensorial grid as illustrated in Fig. 1(b). It is parameterized by two sets of points $\left\{y_{p} \mid p=0, \ldots, P\right\}$ and $\left\{z_{k} \mid k=0, \ldots, K\right\}$ with corresponding intervals $\mathbf{I}(y, p, P)$ and $\mathbf{I}(z, k, K)$ according to (5). A further solution scheme [5] uses a suitable basis set for the expansion of the solution $\Phi^{n}$ from (9). The transverse potential $U_{n}(y, z)$ is then approximated by a set of samples $U_{p k} \equiv U\left(y_{p}, z_{k}\right)$ evaluated at the tensorial grid points as in the FD approach. An analytic representation of the approximated $U_{n}(y, z)$ is given by a stepwise constant function

$U_{n}(y, z) \simeq \sum_{p=0}^{P} \sum_{k=0}^{K} U_{p k} \chi_{p k}(y, z)$,

where $\chi_{p k}(y, z)$ is the characteristic function on the interval $\mathbf{I}(y, p, P) \times \mathbf{I}(z, k, K)$. The position dependence of the transverse effective masses $m_{y} \rightarrow m_{y}\left(x_{n}, y, z\right)$ and $m_{z} \rightarrow$ $m_{z}\left(x_{n}, y, z\right)$ from (9) is treated in the same way as for $U_{n}(y, z)$ in (11). In order to account for Dirichlet boundary conditions, a suitable basis set is given by sine waves having nodes at the boundary of the transverse domain $\left[0, L_{y}\right] \times\left[0, L_{z}\right]$. The series expansion (SE) of the solution $\Phi^{n}(y, z)$ from (9) in terms of sine waves is

$\Phi^{n}(y, z)$

$$
\begin{aligned}
& =\sum_{r=1}^{R} \sum_{s=1}^{S} c_{r s}^{n}\langle y, z \mid r, s\rangle \\
& \equiv \frac{2}{\sqrt{L_{y} L_{z}}} \sum_{r=1}^{R} \sum_{s=1}^{S} c_{r s}^{n} \sin \left(y \frac{r \pi}{L_{y}}\right) \sin \left(z \frac{s \pi}{L_{z}}\right),
\end{aligned}
$$

where $R, S \in \mathbb{N}$ denote cutoffs. These sine waves are orthonormal with respect to the simulation domain $L_{y} \times L_{z}$, i.e.

$$
\begin{aligned}
\left\langle r^{\prime}, s^{\prime} \mid r, s\right\rangle \equiv & \frac{4}{L_{y} L_{z}} \int_{0}^{L_{y}} \int_{0}^{L_{z}} \sin \left(y \frac{r^{\prime} \pi}{L_{y}}\right) \sin \left(z \frac{s^{\prime} \pi}{L_{z}}\right) \\
& \times \sin \left(y \frac{r \pi}{L_{y}}\right) \sin \left(z \frac{s \pi}{L_{z}}\right) d y d z \\
= & \delta_{r, r^{\prime}} \delta_{s, s^{\prime}}
\end{aligned}
$$

The algebraic eigenvalue problem that results when using the ansatz from (12) for the problem stated in (9) is given by

$\mathbf{H}_{n} \mathbf{c}_{n}=\epsilon_{n} \mathbf{c}_{n}$,

where $\mathbf{c}_{n}$ and $\mathbf{H}_{n}$ are defined by

$$
\begin{aligned}
\mathbf{H}_{n}\left(r^{\prime} s^{\prime}, r s\right) \equiv & \left\langle r^{\prime}, s^{\prime}\left|\widehat{\mathbf{H}}_{n}\right| r, s\right\rangle \\
= & \frac{4}{L_{y} L_{z}} \int_{0}^{L_{y}} \int_{0}^{L_{z}} \sin \left(y \frac{r^{\prime} \pi}{L_{y}}\right) \sin \left(z \frac{s^{\prime} \pi}{L_{z}}\right) \\
& \times \widehat{\mathbf{H}}_{n} \sin \left(y \frac{r \pi}{L_{y}}\right) \sin \left(z \frac{s \pi}{L_{z}}\right) d y d z
\end{aligned}
$$
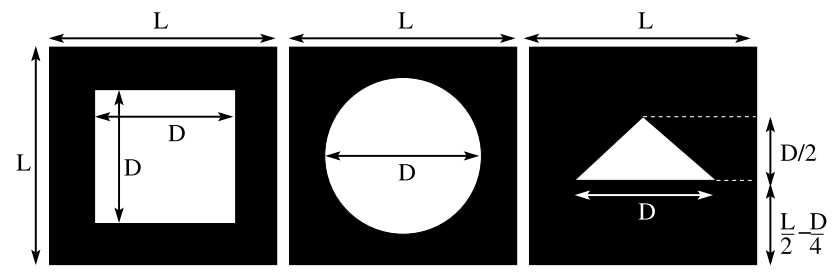

Fig. 2 Square(SQ), circular(CI), and triangular(TR) model potentials used for the comparison of the FD and SE approach described in Sect. 2.2. The dark region denotes a step of $3 \mathrm{eV}$ with respect to the white area which is set to zero. The dimensions are $L_{y}=L_{z}=L=5 \mathrm{~nm}$ and $D=3 \mathrm{~nm}$ and the effective masses are given by $m_{y}=0.19$ and $m_{z}=0.91$

Table 1 Ground state (GS) energies belonging to the three potentials described in Fig. 2. Shown are the results obtained by the FD and SE approach described in Sect. 2.2. The quantity $\mathrm{C}$ denotes the cutoff of the (SE) method whereas for the approximation given in (11) an uniformly distributed grid is chosen with $K=P=Q$. The combinations SQ-FD, SQ-SE, CI-FD, CI-SE, TR-FD, TR-SE are abbreviated by the symbols I to VI respectively. All GS energies are given in $\mathrm{eV}$

\begin{tabular}{llllllll}
\hline C & Q & I & II & III & IV & V & VI \\
\hline 10 & 50 & 0.187 & 0.190 & 0.231 & 0.234 & 0.569 & 0.602 \\
10 & 75 & 0.191 & 0.193 & 0.230 & 0.233 & 0.574 & 0.601 \\
10 & 100 & 0.193 & 0.195 & 0.230 & 0.232 & 0.591 & 0.617 \\
20 & 100 & 0.193 & 0.194 & 0.230 & 0.231 & 0.591 & 0.597 \\
30 & 100 & 0.193 & 0.194 & 0.230 & 0.230 & 0.591 & 0.595 \\
\hline
\end{tabular}

and $\mathbf{c}_{n}(r s)=c_{r s}$, respectively. Note, that as the attention is restricted to square quantum wires, the cutoffs from (12) are both set to a common value $C$, i.e. $R=S=C$. In order to compare the SE and FD methods, three different geometries are considered as described in Fig. 2. The corresponding ground state energies are summarized in Table 1. For the finest grid discretization and largest sine wave expansion both methods agree up to $\simeq 4 \mathrm{meV}$ for all potential shapes.

\subsection{The longitudinal part}

\subsubsection{Steady-state quantum transport equations}

For the description of transport along the well-defined longitudinal direction, the non-equilibrium Green's function (NEGF) formalism is used, since it allows to include scattering mechanisms on a perturbative approach. Starting from the continuous formulation of the steady-state quantum transport equations, the corresponding expression in the coupled mode expansion will be derived. A detailed description of the electron-phonon interaction will be given in Sect. 2.5.

The solution variables of the steady-state Dyson and Keldysh equation are the retarded $\left(G^{R}\right)$ and lesser $\left(G^{<}\right)$ 
Green's function [20, 21]:

$$
\begin{aligned}
& \int d \mathbf{r}_{1}\left[(E-H(\mathbf{r})) \delta\left(\mathbf{r}-\mathbf{r}_{1}\right)-\Sigma^{R}\left(\mathbf{r}, \mathbf{r}_{1}, E\right)\right] \\
& \quad \times G^{R}\left(\mathbf{r}_{1}, \mathbf{r}^{\prime}, E\right)=\delta\left(\mathbf{r}-\mathbf{r}^{\prime}\right)
\end{aligned}
$$

$$
\begin{aligned}
G^{<}\left(\mathbf{r}, \mathbf{r}^{\prime}, E\right)= & \int d \mathbf{r}_{1} \int d \mathbf{r}_{2} G^{R}\left(\mathbf{r}, \mathbf{r}_{1}, E\right) \\
& \times \Sigma^{<}\left(\mathbf{r}_{1}, \mathbf{r}_{2}, E\right) G^{A}\left(\mathbf{r}_{1}, \mathbf{r}^{\prime}, E\right),
\end{aligned}
$$

where $H(\mathbf{r})$ is the Hamiltonian of the system given in (1) and $\Sigma\left(\mathbf{r}, \mathbf{r}^{\prime}, E\right)$ the self-energy, containing the electronphonon interaction $\Sigma_{\text {int }}\left(\mathbf{r}, \mathbf{r}^{\prime}, E\right.$ ) (whereas the Hartree potential is already included in the Hamiltonian) as well as the boundary conditions $\Sigma_{b c}\left(\mathbf{r}, \mathbf{r}^{\prime}, E\right)$ :

$$
\Sigma\left(\mathbf{r}, \mathbf{r}^{\prime}, E\right)=\Sigma_{i n t}\left(\mathbf{r}, \mathbf{r}^{\prime}, E\right)+\Sigma_{b c}\left(\mathbf{r}, \mathbf{r}^{\prime}, E\right) .
$$

\subsubsection{Coupled mode expansion}

For devices with a well-defined longitudinal direction, it has been shown that the coupled mode approach yields the same results as a real-space approach, yet decreasing the computational burden significantly [22, 23].

All quantities of interest, i.e. the various Green's functions and self-energies can now be expressed in the basis functions as defined in (8):

$G^{R}\left(\mathbf{r}, \mathbf{r}^{\prime}, E\right)=\sum_{i, j} G_{i j}^{R}\left(x_{n}, x_{m}, E\right) \Phi_{i}^{* n}(y, z) \Phi_{j}^{m}\left(y^{\prime}, z^{\prime}\right)$,

where $G_{i j}^{R}\left(x_{n}, x_{m}, E\right)$ is the solution of the Dyson equation formulated in mode-space

$$
\begin{aligned}
\sum_{i, n^{\prime}}( & E \delta_{j i} \delta_{n n^{\prime}}-\int d y d z \Phi_{j}^{* n}(y, z) H(\mathbf{r}) \Phi_{i}^{n^{\prime}}(y, z) \\
& \left.-\int d y d z \int d y^{\prime} d z^{\prime} \Phi_{j}^{* n}(y, z) \Sigma^{R}\left(\mathbf{r}, \mathbf{r}^{\prime}\right) \Phi_{i}^{n^{\prime}}\left(y^{\prime}, z^{\prime}\right)\right) \\
& \times G_{i k}^{R}\left(x_{n^{\prime}}, x_{m}, E\right) \\
= & \sum_{i, n^{\prime}}\left[E \delta_{j i} \delta_{n n^{\prime}}-H_{j i}\left(x_{n}, x_{n^{\prime}}\right)-\Sigma_{j i}^{R}\left(x_{n}, x_{n^{\prime}}, E\right)\right] \\
& \times G_{i k}^{R}\left(x_{n^{\prime}}, x_{m}, E\right)=\delta_{j k} \delta\left(x_{n}-x_{m}\right) .
\end{aligned}
$$

Equation (20) is obtained by inserting (19) into (16), multiplying with $\Phi_{j}^{* n}(y, z)$ from the left and $\Phi_{i}^{n^{\prime}}\left(y^{\prime}, z^{\prime}\right)$ from the right and finally integrating over $\mathbf{r}$ and $\mathbf{r}^{\prime}$.

Using the same procedure, the expressions for the selfenergy in real-space and mode space can be obtained. Assuming that the real-space expression can be written as

$$
\begin{aligned}
& \Sigma^{R}\left(\mathbf{r}, \mathbf{r}^{\prime}, E\right) \\
& \quad=C\left(\mathbf{r}, \mathbf{r}^{\prime}\right) G^{R}\left(\mathbf{r}, \mathbf{r}^{\prime}, E\right) \\
& \quad=\sum_{i, j} C\left(\mathbf{r}, \mathbf{r}^{\prime}\right) G_{i j}^{R}\left(x_{n}, x_{m}, E\right) \Phi_{i}^{* n}(y, z) \Phi_{i}^{m}\left(y^{\prime}, z^{\prime}\right),
\end{aligned}
$$

the self-energy in mode-space is defined by

$$
\begin{aligned}
& \Sigma_{i j}^{R}\left(x_{n}, x_{m}, E\right) \\
& =\sum_{k, l}\left(\int d y d z \int d y^{\prime} d z^{\prime}\right. \\
& \quad \times \Phi_{i}^{* n}(y, z) \Phi_{k}^{* n}(y, z) C\left(\mathbf{r}, \mathbf{r}^{\prime}\right) \Phi_{l}^{m}\left(y^{\prime}, z^{\prime}\right) \Phi_{j}^{m}\left(y^{\prime}, z^{\prime}\right) \\
& \left.\quad \times G_{k l}^{R}\left(x_{n}, x_{m}, E\right)\right)=\sum_{k, l} C_{i j k l} G_{k l}^{R}\left(x_{n}, x_{m}, E\right)
\end{aligned}
$$

Note that the exact form of the self-energy depends on the respective scattering mechanism. The form written in (21), which applies to the intravalley acoustic electron-phonon interaction, was chosen as an example in order to illustrate the transformation behavior.

The boundary self-energies are not transformed but directly computed in mode-space [24-26].

The advantage of the coupled mode approach lies in the reduction of the matrix size for the transport problem, since the square of the number of modes $N_{M}^{2}$ needed in the simulations is much smaller than the number of grid points $\left(N_{y}, N_{z}\right)$ in the transverse direction: $N_{M}^{2} \ll N_{y} \cdot N_{z}$.

On the other hand, one of the major shortcomings of this approach is that only transport along crystal direction $\langle 100\rangle$ is possible for nanowires.

\subsection{Nonparabolicity}

In order to improve the EMA, some of the underlying prerequisites have to be reconsidered in a more general way. An important point is that the wave function belonging to the Hamiltonian including the crystal potential and the external perturbation can be well described within a single energy band of the crystal band structure, i.e. the lowest silicon conduction band

$\Psi=\sum_{\mu, \mathbf{k}} c_{\mu}(\mathbf{k}) \phi_{\mu}(\mathbf{k}, \mathbf{r}) \simeq \sum_{\mathbf{k}} c_{\mathrm{CB}}(\mathbf{k}) \phi_{\mathrm{CB}}(\mathbf{k}, \mathbf{r})$.

The latter simplification is related to the assumption that the external perturbation is too weak to induce a transition from one band to another. The corresponding Schrödinger equation when using the approximate series expansion from (23) reads

$\sum_{\mathbf{k}} c_{\mathrm{CB}}(\mathbf{k})\left[-\frac{\hbar^{2}}{2 m_{e}}+V(\mathbf{r})+U(\mathbf{r})\right] \phi_{\mathrm{CB}}(\mathbf{k}, \mathbf{r})=E \Psi(\mathbf{r})$ 
and can be rewritten as

$\left[\epsilon_{\mathrm{CB}}(-i \nabla)+U(\mathbf{r})\right] \Psi(\mathbf{r})=E \Psi(\mathbf{r})$

where $V(\mathbf{r})$ denotes the crystal potential, $U(\mathbf{r})$ the external perturbation, and $\epsilon_{\mathrm{CB}}(\mathbf{k})$ is the conduction band. The EMA dispersion relies on a second order expansion of $\epsilon_{\mathrm{CB}}(\mathbf{k})$ in (25) around the minimum. Sticking to the single band picture, several efforts have been made [1, 3-12] in order to bridge the gap between the simple parabolic dispersion contained in the EMA and the shape of the corresponding conduction band. In this work a modification is provided by a nonparabolic $[6,7]$ dispersion of the form

$\left(E-\epsilon_{c}\right)\left[1+\alpha\left(E-\epsilon_{c}\right)\right] \equiv \frac{\hbar^{2}}{2} \mathbf{k}^{\mathrm{T}} \mathbf{M} \mathbf{k}$

and consequently

$E(\mathbf{k})=\frac{1}{2 \alpha}\left[\sqrt{1+4 \alpha \frac{\hbar^{2}}{2} \mathbf{k}^{\mathrm{T}} \mathbf{M} \mathbf{k}}-1\right]+\epsilon_{c}$,

where $\alpha$ is referred to as the nonparabolicity coefficient having the dimension of an inverse energy and $\epsilon_{c}=1.12 \mathrm{eV}$. The replacement of $\mathbf{k}$ by $-i \nabla$ yields a Schrödinger equation of the form

$$
\begin{aligned}
& {\left[\frac{1}{2 \alpha}\left(\sqrt{1-4 \alpha \frac{\hbar^{2}}{2} \nabla^{\mathrm{T}} \mathbf{M} \nabla}-1\right)+\epsilon_{c}\right.} \\
& \quad+U(\mathbf{r})] \Psi(\mathbf{r})=\epsilon \Psi(\mathbf{r}) .
\end{aligned}
$$

In order to decouple the expression given in (28) as described in Sect. 2.1 and consequently recover the starting point for the transport framework used in this work, some assumptions have to made which are related to the presence of a one-dimensional transverse confinement as it is the case in quantum wires. In the following, the derivation of such a simplified expression is closely related to Ref. [2]. The impact of hard wall conditions at the boundaries of a domain $[0, D] \times[0, D]$ on the Schrödinger problem given in (28) for $U \equiv 0$ can be investigated by means of the resulting energy spectrum which in the case of a diagonal effective mass tensor $\mathbf{M}$ is given by

$\epsilon\left(n, m, k_{x}, \alpha\right) \equiv \frac{1}{2 \alpha}\left[\sqrt{1+4 \alpha\left(\epsilon_{\|}+\epsilon_{\perp}\right)}-1\right]+\epsilon_{c}$

with $n, m=1,2, \ldots, \epsilon_{\|}\left(k_{x}\right) \equiv \hbar^{2} k_{x}^{2} /\left(2 m_{x}\right)$, and $\epsilon_{\perp}(n, m) \equiv$ $\hbar^{2} \pi^{2}\left(n^{2} / m_{y}+m^{2} / m_{z}\right) /\left(2 D^{2}\right)$. The assumption that the transverse confinement prevails against the longitudinal one, i.e. $\epsilon_{\|} \ll \epsilon_{\perp}$, yields

$\epsilon\left(n, m, k_{x}, \alpha\right)=\frac{1}{2 \alpha}\left[\sqrt{1+4 \alpha \epsilon_{\perp}}-1\right]+\epsilon_{c}$

$$
+\frac{\epsilon_{\|}}{\sqrt{1+4 \alpha \epsilon_{\perp}}}+O\left[\left(\frac{\epsilon_{\|}}{\epsilon_{\perp}}\right)^{2}\right] \text {. }
$$

With the abbreviations $\beta\left(\alpha, \epsilon_{\perp}\right) \equiv \sqrt{1+4 \alpha \epsilon_{\perp}}$ and $\epsilon^{\mathrm{NP}}(n, m, \alpha) \equiv\left[\sqrt{1+4 \alpha \epsilon_{\perp}}-1\right] /(2 \alpha)+\epsilon_{c}$ the spectrum given in (30) can be approximated by

$\epsilon\left(n, m, k_{x}, \alpha\right) \simeq \epsilon^{\mathrm{NP}}(n, m, \alpha)+\frac{\epsilon_{\|}}{\beta\left(\alpha, \epsilon_{\perp}\right)}$.

For simplicity, the abbreviations $\beta_{c} \equiv \beta\left[\alpha, \epsilon_{\perp}(1,1)\right]$ and $\epsilon_{c}^{\mathrm{NP}} \equiv \epsilon^{\mathrm{NP}}(1,1, \alpha)$ will be used. Note that the mass $m_{x}$ on the right hand side of (31) is effectively renormalized (increased) due to the lateral confinement according to

$m_{x} \rightarrow m_{x} \beta\left(\alpha, \epsilon_{\perp}\right)$

In the case of the conduction band edge the quantity $\widetilde{m}_{x} \equiv$ $m_{x} \beta_{c}$ is referred to as the conduction mass. Based on (31), the Schrödinger problem given in (6) is modified to

$\left(-\frac{\hbar^{2}}{2 \widetilde{m}_{x}} \frac{\partial^{2}}{\partial x^{2}}+\widehat{\mathbf{H}}_{n}^{\mathrm{NP}}\right) \Psi(\mathbf{r})=E \Psi(\mathbf{r})$,

where

$$
\begin{aligned}
\widehat{\mathbf{H}}_{n}^{\mathrm{NP}} \equiv & \frac{1}{2 \alpha}\left[\sqrt{1-4 \alpha \frac{\hbar^{2}}{2}\left(\frac{1}{m_{y}} \frac{\partial^{2}}{\partial y^{2}}+\frac{1}{m_{z}} \frac{\partial^{2}}{\partial z^{2}}\right)}-1\right] \\
& +U_{n}(y, z) .
\end{aligned}
$$

The change of the operator ordering in (34) compared to (7) is justified by the requirement that $\left\{m_{x}, m_{y}, m_{z}\right\}$ are position independent as it is assumed for the rest of the work. The spectrum of the Hamiltonian (34) can be computed by means of the SE method introduced in Sect. 2.2. For the determination of the NP coefficients $\alpha$ and corresponding renormalized conduction masses $\tilde{m}_{x}$ the TB formalism will be used. The band structures of square silicon quantum wires having a channel grown along the $\langle 100\rangle$ direction are computed by a TB [15] simulator for a distinct set of wire widths $D[\mathrm{~nm}] \in\{2.04,2.44,2.85,3.26$, $3.53,3.94,4.34\}$. The TB band structure for the case $D=$ $3.26 \mathrm{~nm}$ is shown in Fig. 3 together with the corresponding dispersions $\epsilon_{c}^{\mathrm{NP}}+\epsilon_{\|} / \beta_{c}$ from (31) for one of the four unprimed valleys $\Delta_{4}$, i.e. $\left(m_{x}, m_{y}, m_{z}\right) / m_{e}=(0.19,0.91$, $0.19)$. In addition, the EMA case $\epsilon_{c}^{\mathrm{EMA}}+\epsilon_{\|}$is plotted, where $\epsilon_{c}^{\mathrm{EMA}} \equiv \epsilon^{\mathrm{NP}}(1,1,0)$. The condition $\epsilon_{c}^{\mathrm{NP}}-\epsilon_{c}^{\mathrm{TB}}=0$ is finally used in order to determine the NP coefficient $\alpha$, where $\epsilon_{c}^{\mathrm{TB}}$ is the TB conduction band $E^{\mathrm{TB}}\left(k_{x}\right)$ evaluated at the $\Gamma$ point, i.e. at $k_{x}=0$. Table 2 summarizes the quantities $\epsilon_{c}^{\mathrm{EMA}}, \epsilon_{c}^{\mathrm{TB}}, \alpha, \tilde{m}_{x}$, as well as $m_{x}^{\mathrm{TB}} \equiv \hbar^{2} /\left(\partial^{2} E^{\mathrm{TB}} / \partial^{2} k_{x}\right)$ evaluated at the $\Gamma$ point. The conduction masses extracted from the TB and NP band structure are found to be close for the case of the unprimed valleys $\Delta_{4}$. Note that in the following the NP model is adopted exclusively for the $\Delta_{4}$ valleys as 


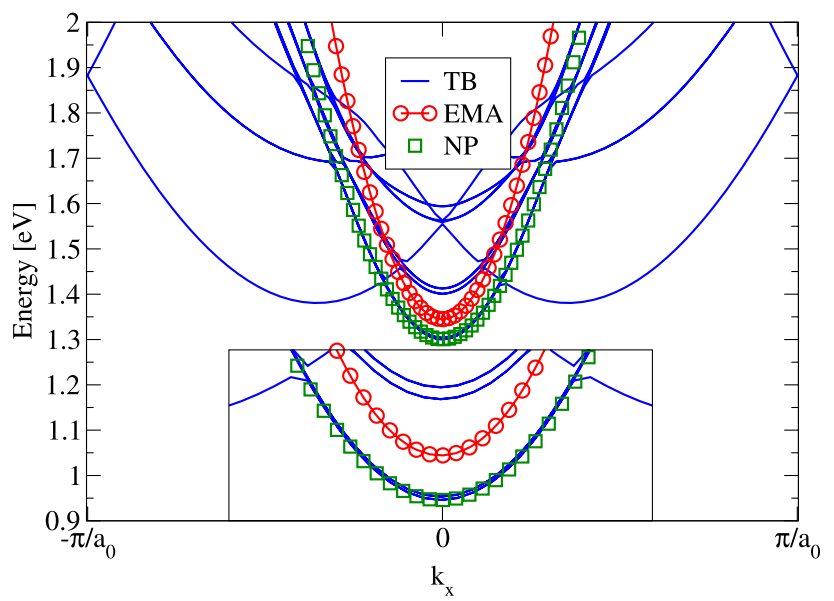

Fig. 3 TB dispersion of a square silicon nanowire grown along the $\langle 100\rangle$ direction having a width of $D=3.26 \mathrm{~nm}$. The length of the wire unit cell is $a_{0}=0.543 \mathrm{~nm}$. In addition, the dispersion $\epsilon_{c}^{\mathrm{NP}}+\epsilon_{\|} / \beta_{c}$ from (31) is plotted together with the EMA case $\epsilon_{c}^{\text {EMA }}+\epsilon_{\|}$. The inset highlights the agreement between $\epsilon_{c}^{\mathrm{NP}}+\epsilon_{\|} / \beta_{c}$ and the TB conduction band

Table 2 Conduction band edges, conduction masses, and NP coefficients as introduced in Sect. 2.4 belonging to a series of square silicon quantum wires of width $D$. Lengths are given in $\mathrm{nm}$, energies in $\mathrm{eV}$, NP coefficients in $\mathrm{eV}^{-1}$, and masses in units of $m_{e}$

\begin{tabular}{llllll}
\hline $\mathrm{D}$ & $\epsilon_{c}^{\mathrm{EMA}}$ & $\epsilon_{c}^{\mathrm{TB}}$ & $\alpha$ & $\tilde{m}_{x}$ & $m_{x}^{\mathrm{TB}}$ \\
\hline 2.04 & 1.694 & 1.512 & 1.19 & 0.367 & 0.370 \\
2.44 & 1.522 & 1.412 & 1.30 & 0.334 & 0.308 \\
2.85 & 1.414 & 1.346 & 1.37 & 0.306 & 0.294 \\
3.26 & 1.345 & 1.301 & 1.36 & 0.283 & 0.267 \\
3.53 & 1.312 & 1.279 & 1.33 & 0.270 & 0.260 \\
3.94 & 1.274 & 1.252 & 1.26 & 0.253 & 0.254 \\
4.34 & 1.247 & 1.233 & 1.14 & 0.239 & 0.242 \\
\hline
\end{tabular}

the influence of the primed valleys is found to be negligible especially for strong lateral confinements.

\subsection{Electron-phonon scattering}

\subsubsection{The lesser scattering self-energy}

Since both electron and phonons are described by their respective wave functions, scattering in quantum mechanics is generally a spatially correlated phenomenon. However, in order to decrease the computational burden, it will be approximated as local in space. In this section, all equations are written in their most general continuous form. In order to calculate the expression in the coupled mode expansion, the transformation described in (22) is used.

It is assumed that the phonon system remains in equilibrium and that the phonon wave functions can be approximated by their bulk counterparts. Since silicon is a non-polar semiconductor, deformation potential theory can be applied in the formulation of the perturbative Hamiltonian [28], which is used to calculate the electron-phonon matrix elements $\left|M_{q}\right|^{2}$.

Then, within the self-consistent Born approximation, the self-energy for the electron-phonon interaction is

$\Sigma^{<}=D^{<} G^{<}$,

with the free phonon lesser Green's function $D^{<}$[27]. For the steady-state case, (35) can be written as

$$
\begin{aligned}
\Sigma^{<}\left(\mathbf{r}, \mathbf{r}^{\prime}, E\right)= & \int \frac{d \mathbf{q}}{(2 \pi)^{3}} e^{i \mathbf{q}\left(\mathbf{r}-\mathbf{r}^{\prime}\right)}\left|M_{q}\right|^{2} \\
& \times\left(N_{q} G^{<}\left(\mathbf{r}, \mathbf{r}^{\prime}, E-\hbar \omega_{q}\right)\right. \\
& \left.+\left(N_{q}+1\right) G^{<}\left(\mathbf{r}, \mathbf{r}^{\prime}, E+\hbar \omega_{q}\right)\right)
\end{aligned}
$$

\subsubsection{The retarded scattering self-energy}

The retarded self-energy is generally written as

$$
\begin{aligned}
\Sigma^{R}\left(\mathbf{r}, \mathbf{r}^{\prime}, E\right) \\
=\frac{1}{2}\left(\Sigma^{>}\left(\mathbf{r}, \mathbf{r}^{\prime}, E\right)-\Sigma^{<}\left(\mathbf{r}, \mathbf{r}^{\prime}, E\right)\right) \\
\quad+i P \int \frac{d E^{\prime}}{2 \pi} \frac{\Sigma^{>}\left(\mathbf{r}, \mathbf{r}^{\prime}, E^{\prime}\right)-\Sigma^{<}\left(\mathbf{r}, \mathbf{r}^{\prime}, E^{\prime}\right)}{E-E^{\prime}},
\end{aligned}
$$

where $P \int d E^{\prime}$ is the principal part of the integration. It consists of two parts:

1. The term on the first line of (37) is anti-Hermitian and responsible for the dephasing.

2. The term with the principal part on the second line of (37) is Hermitian and yields a renormalization of the energy.

The solution variables of the Dyson and Keldysh equation are the retarded and the lesser Green's functions $\left(G^{R}, G^{<}\right)$. In order to calculate $\Sigma^{>}$in (37), which depends on $G^{>}$, the following relation can be applied:

$G^{>}(E)=G^{R}(E)-G^{A}(E)+G^{<}(E)$.

However, the retarded self-energy can be calculated directly from the solution variables $\left(G^{R}, G^{<}\right)$, without an explicit calculation of $G^{>}$:

$$
\begin{aligned}
\Sigma^{R}\left(\mathbf{r}, \mathbf{r}^{\prime}, E\right)= & \int \frac{d \mathbf{q}}{(2 \pi)^{3}} e^{i \mathbf{q}\left(\mathbf{r}-\mathbf{r}^{\prime}\right)}\left|M_{q}\right|^{2} \\
& \times\left(\left(N_{q}+1\right) G^{R}\left(\mathbf{r}, \mathbf{r}^{\prime}, E-\hbar \omega_{q}\right)\right. \\
& +N_{q} G^{R}\left(\mathbf{r}, \mathbf{r}^{\prime}, E+\hbar \omega_{q}\right) \\
& +\frac{1}{2}\left(G^{<}\left(\mathbf{r}, \mathbf{r}^{\prime}, E-\hbar \omega_{q}\right)\right.
\end{aligned}
$$




$$
\begin{aligned}
& \left.-G^{<}\left(\mathbf{r}, \mathbf{r}^{\prime}, E+\hbar \omega_{q}\right)\right) \\
& +i P \int \frac{d E^{\prime}}{2 \pi} \frac{G^{<}\left(\mathbf{r}, \mathbf{r}^{\prime}, E-E^{\prime}\right)}{E^{\prime}-\hbar \omega_{q}} \\
& \left.-\frac{G^{<}\left(\mathbf{r}, \mathbf{r}^{\prime}, E-E^{\prime}\right)}{E^{\prime}+\hbar \omega_{q}}\right) .
\end{aligned}
$$

A derivation of (39) is given in [29]. Note that in (39) above, the term with the principal part integral contains only a fraction of the original Hermitian term in (37). It is therefore expected, that neglecting the principal part integral in (39) instead of (37) results in a much smaller error on both the density and the current. A detailed discussion of both approximations compared to the full expression is given in Sect. 3.3.

\subsubsection{Intravalley acoustic phonon scattering}

Assuming that $\hbar \omega_{q} \ll k_{B} T \Rightarrow E \pm \hbar \omega_{q} \approx E$, intravalley acoustic phonon scattering becomes an elastic interaction and the phonon distribution function can be approximated by

$N_{q}+1 \approx N_{q} \approx \frac{k_{B} T}{\hbar \omega_{q}}$.

Taking a linear dispersion relation $\hbar \omega_{q}=c_{s} q$, the corresponding electron-phonon matrix element can be approximated by

$\left|M_{q}\right|^{2} \approx \frac{\hbar \Xi^{2} q}{2 \rho c_{s}}$

Then the term $\left|M_{q}\right|^{2} \cdot N_{q}$ in (36) becomes independent of the phonon wave vector $q$, and therefore the self-energy is approximated as local in space:

$\Sigma_{v}^{<}\left(\mathbf{r}, \mathbf{r}^{\prime}, E\right)=\frac{\Xi^{2} k_{B} T}{\rho c_{s}^{2}} G_{v}^{<}\left(\mathbf{r}, \mathbf{r}^{\prime}, E\right) \delta\left(\mathbf{r}-\mathbf{r}^{\prime}\right)$.

The mass density of silicon is $\rho=2.329 \mathrm{~g} / \mathrm{cm}^{3}$, the speed of sound in silicon is $c_{s}=9.04 \cdot 10^{5} \mathrm{~cm} / \mathrm{s}$ and for the deformation potential $\Xi=14.6 \mathrm{eV}$ is used as proposed in [30].

\subsubsection{Intervalley phonon scattering}

Assuming that both the phonon energy and the matrix element can be regarded as being independent of the phonon wave vector, the lesser self-energy for intervalley phonon scattering is approximated as diagonal in space too.

The electron-phonon matrix elements can be written as

$\left|M_{j}\right|^{2}=\frac{\hbar^{2}\left(D_{t} K_{j}\right)^{2}}{2 \rho \hbar \omega_{j}}$,

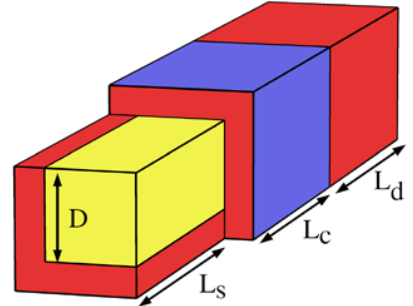

Fig. 4 A triple gate square silicon nanowire having a channel grown along the $\langle 100\rangle$ direction which is surrounded by a $1 \mathrm{~nm}$ thick oxide layer. The source and drain regions are n-doped with a concentration of $10^{20} \mathrm{~cm}^{-3}$ while the channel is undoped. The lengths of the source, channel, and drain regions are given by $L_{s}=9.7 \mathrm{~nm}, L_{c}=15 \mathrm{~nm}$, and $L_{d}=9.7 \mathrm{~nm}$ respectively and the wire width is denoted by $D$

where $D_{t} K_{j}$ is the coupling constant and $\hbar \omega_{j}$ the phonon energy of the respective intervalley scattering mechanism. The parameters are taken from [28].

The lesser self energies for valley $v$ are given by a sum over all valleys, using the appropriate selection rule $\left(s_{v, v^{\prime}}=\right.$ $\delta_{v, v^{\prime}}$ for g-type, and $s_{v, v^{\prime}}=2\left(1-\delta_{v, v^{\prime}}\right)$ for f-type intervalley scattering):

$$
\begin{aligned}
\Sigma_{j, v}^{<}\left(\mathbf{r}, \mathbf{r}^{\prime}, E\right)= & \frac{\hbar^{2}\left(D_{t} K_{j}\right)^{2}}{2 \rho \hbar \omega_{j}} \sum_{v^{\prime}=1}^{3}\left(N_{j} G_{v^{\prime}}^{<}\left(\mathbf{r}, \mathbf{r}^{\prime}, E-\hbar \omega_{j}\right)\right. \\
& +\left(N_{j}+1\right) G_{v^{\prime}}^{<}\left(\mathbf{r}, \mathbf{r}^{\prime}, E\right. \\
& \left.\left.+\hbar \omega_{j}\right)\right) s_{v, v^{\prime}} \delta\left(\mathbf{r}-\mathbf{r}^{\prime}\right)
\end{aligned}
$$

\subsection{Density and current calculation}

For both the carrier density and the current, we basically have three contributions: coherent terms from the source/drain contacts (boundary terms) and an incoherent term from the electron-phonon interaction.

$G^{<}=G^{R}\left(\Sigma_{S}^{<}+\Sigma_{D}^{<}+\Sigma_{\text {int }}^{<}\right) G^{A}$,

$$
\begin{aligned}
n\left(x_{n}, y, z\right)= & -i \sum_{v, \sigma} \sum_{i j} \int \frac{d E}{2 \pi} \\
& \times G_{i j}^{<, v}\left(x_{n}, x_{n}, E\right) \Phi_{i}^{* n}(y, z) \Phi_{j}^{* n}(y, z)
\end{aligned}
$$

$$
\begin{aligned}
I\left(x_{n}\right)= & -\frac{e}{\hbar} \sum_{v, \sigma} \sum_{i j} \int \frac{d E}{2 \pi} \\
& \times\left(2 \operatorname{Re}\left(H_{i j}^{v}\left(x_{n}, x_{n+1}\right) G_{j i}^{<, v}\left(x_{n+1}, x_{n}\right)\right)\right) .
\end{aligned}
$$

In (46) and (47) $v$ and $\sigma$ are the valley and spin indices. 


\section{Simulation results}

\subsection{Test framework}

The impact of scattering and nonparabolicity is investigated by means of a square silicon quantum wire transistor as described in Fig. 4 which serves as basis for all current computations in the rest of the work. The threshold voltage $V_{\mathrm{T}}$ and the ON-current $I_{\mathrm{ON}}$ are defined similar to Ref. [9] by

$I_{\mathrm{DS}}\left(V_{\mathrm{GS}}=V_{\mathrm{T}}, V_{\mathrm{DS}}\right)=100 \mathrm{nA}$

and

$I_{\mathrm{ON}}=I_{\mathrm{DS}}\left(V_{\mathrm{T}}+0.3 V, V_{\mathrm{DS}}\right)$,

where $I_{\mathrm{DS}}, V_{\mathrm{GS}}$, and $V_{\mathrm{DS}}$ denote the drain current, gate voltage, and source-to-drain bias respectively.

\subsection{Nonparabolicity}

The framework presented in Sect. 2.4 aims at reducing the discrepancy between the currents obtained by the EMA and a fully atomistic TB approach [15]. In order to quantitatively investigate the improvement delivered by the NP model a set of transfer characteristics related to the diameters $D[\mathrm{~nm}] \in\{2.04,2.44,2.85,3.26,3.53,3.94,4.34\}$ are computed with the TB, EMA, and NP formalism. The resulting $V_{\mathrm{T}}$ and $I_{\mathrm{ON}}$ are extracted by means of (48) and (49) at a source-to-drain bias of $0.6 \mathrm{~V}$ and plotted in Fig. 5. It can be seen that the $V_{\mathrm{T}}$ obtained by the NP model is notably closer to TB data than the results obtained with the EMA. Similar observations have been reported for the case of cylindrical [12] and square [9] silicon nanowire transistors. An improvement for the $I_{\mathrm{ON}}$ is evident as well but not as apparent as in the $V_{\mathrm{T}}$ case. Noticeable are the similarities between the $I_{\mathrm{ON}}$ obtained by the EMA and the NP model for $\widetilde{m}_{x}=0.19 m_{e}$ showing that the modified conduction mass is mainly responsible for the ON-current shift resulting from the NP simulation. On the other hand, the conduction mass has a minor influence on the threshold voltage, where the shift of $V_{\mathrm{T}}$ can be attributed to the shift of the conduction band edge between the EMA, NP, and TB cases. The latter observations are of course restricted to the device considered in this section.

\subsection{Approximations of the retarded electron-phonon self-energy}

When calculating the full retarded self-energy of an inelastic interaction, a principal part integration over the complete energy range must be carried out for each energy sampling point, as shown in (37). In order to decrease the computational burden, different approximations for the retarded selfenergy can be made [30-32]. In this section, the validity of these approximations under different bias conditions is analyzed by comparing them to results calculated with the full retarded self-energy.

The different definitions of $\Sigma_{\text {int }}^{R}\left(\mathbf{r}, \mathbf{r}^{\prime}, E\right)$ used here are given by:

- Approximation I: Equation (37) without the last line (i.e. neglecting the princ. part integral)

- Approximation II: Equation (39) without the last line (i.e. neglecting the princ. part integral)

- Full: the full expression in (39).

Both approximations of the retarded self-energy respect current conservation, which is shown in the Appendix for the sake of completeness. As already mentioned in Sect. 2.5.2, Approximation I only includes the anti-Hermitian term while the Hermitian term is neglected completely. In contrast to this, Approximation II not only contains the anti-Hermitian term, but also a fraction of the Hermitian term.

In Fig. 7 the $I_{\mathrm{DS}} V_{\mathrm{G}}$ curves are shown for all 3 versions of $\Sigma_{\text {int }}^{R}\left(\mathbf{r}, \mathbf{r}^{\prime}, E\right)$. Approximation I yields an underestimation of the subtreshold current up to $80 \%$ when compared to Full version. However the discrepancy between the two current curves is decreasing for $V_{\mathrm{G}}>V_{\mathrm{T}}$ and is within an range of $5 \%$ for $I_{\mathrm{ON}}$. This behavior can be explained by the impact of the neglected energy renormalization on the effective barrier height. In the subtreshold voltage regime, the current increases exponentially as the barrier height decreases. Therefore even a small change in the effective barrier height has a strong impact on the current. Once the barrier height becomes comparable to $k_{B} T$, as it is the case for $I_{\mathrm{ON}}$, a small renormalization of the barrier has almost no effect on the current.

The resulting current of Approximation II is in excellent agreement with the current of the Full version throughout the whole voltage regime. The deviation is less than $1 \%$, i.e. it lies within the range of the numerical tolerance for the current calculation.

In Fig. 6 the lowest subbands and the spectral electron densities taken at $x=17.2 \mathrm{~nm}$ are shown. Both the subbands and the spectral density of Approximation I deviate from the other versions, whereas the data of Approximation II is almost identical to the Full version in the gate region. However there is still a difference in the electrostatic solution in the source-drain extensions.

\subsection{Combination of nonparabolicity and scattering}

In this section, the results of simulations incorporating both nonparabolicity and scattering (NPSC) are compared to data from simulation containing only NP, SC or EMA. For all the $\mathrm{SC}$ data in this section, Approximation II is used for the retarded self-energy, since it yields the accurate electrostatic 


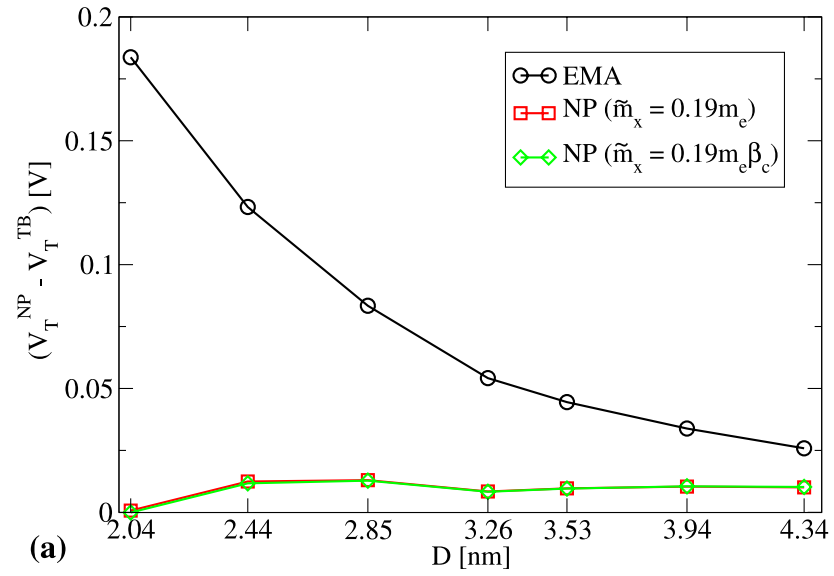

Fig. 5 Dependence of the errors $\left(V_{\mathrm{T}}^{\mathrm{NP}}-V_{\mathrm{T}}^{\mathrm{TB}}\right)(\mathbf{a})$ and $\left(I_{\mathrm{ON}}^{\mathrm{NP}}-\right.$ $\left.I_{\mathrm{ON}}^{\mathrm{TB}}\right) / I_{\mathrm{ON}}^{\mathrm{TB}}(\mathbf{b})$ on the wire width $D$. Plotted are the results obtained with the set of NP parameters summarized in Table 2. In particular, the squares and diamonds are for the same set of coefficients $\alpha$ whereas for the latter curve $\widetilde{m}_{x}=0.19 m_{e}$ has been used instead of $\widetilde{m}_{x}=0.19 m_{e} \beta_{c}$.

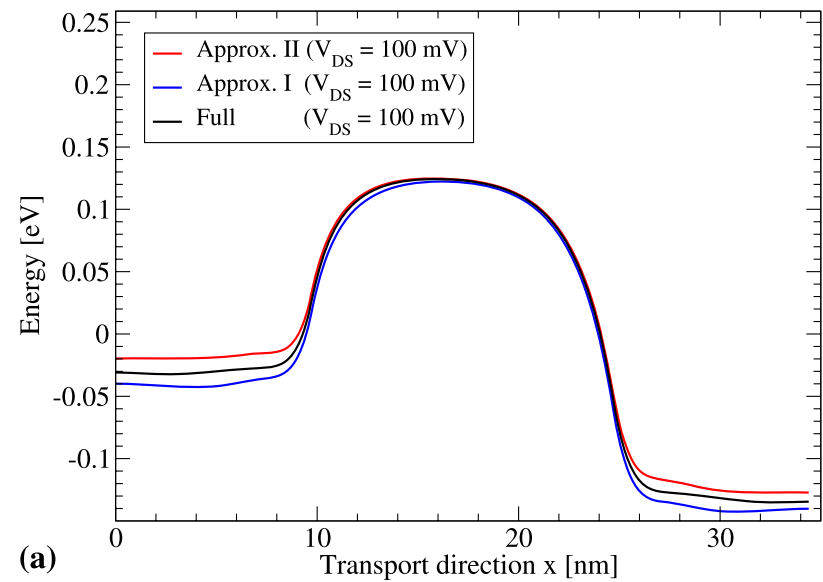

Fig. 6 (a) The lowest subband at $V_{\mathrm{G}}=0.3 \mathrm{~V}$ is shown for the different approximations of the retarded self-energy compared to the full solution. The spectral electron density in the middle of the device (cut

solution and current, but decreases the computational burden, as demonstrated in Sect. 3.3. The results of Figs. 8 and 9 are summarized in Table 3.

The threshold voltage $V_{\mathrm{T}}$ is mainly affected by the modification of the band structure, i.e. the inclusion of NP, for both source-to-drain biases. The effect of SC on $V_{\mathrm{T}}$ is on the order of $1 \%$ and therefore negligible.

On the other hand, the change in $I_{\mathrm{ON}}$ is mainly due to scattering while the modification of the conduction mass resulting from the NP model has a minor impact only. It is interesting to note that for the ON-current, the single methods SC and NP do not add up to the result of the combination NPSC. This can be explained in the following way: Bandstructure effects beyond the EMA approach become impor-

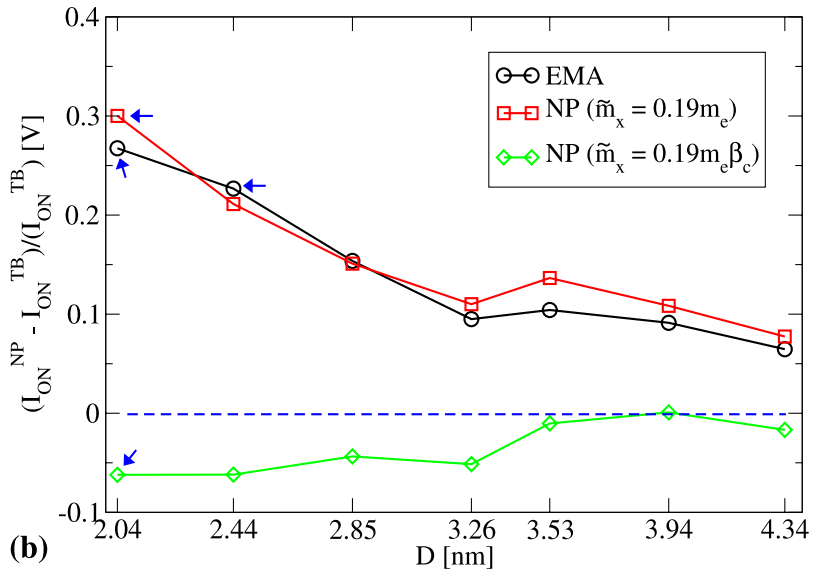

The arrows indicate points for which the transfer characteristics do not converge up to the desired gate voltage. For these points a parabolic fit to the data above $V_{\mathrm{T}}$ is used to extrapolate the $I_{\mathrm{ON}}$ according to (49). The remaining $V_{\mathrm{T}}$ and $I_{\mathrm{ON}}$ are computed by means of a spline interpolation

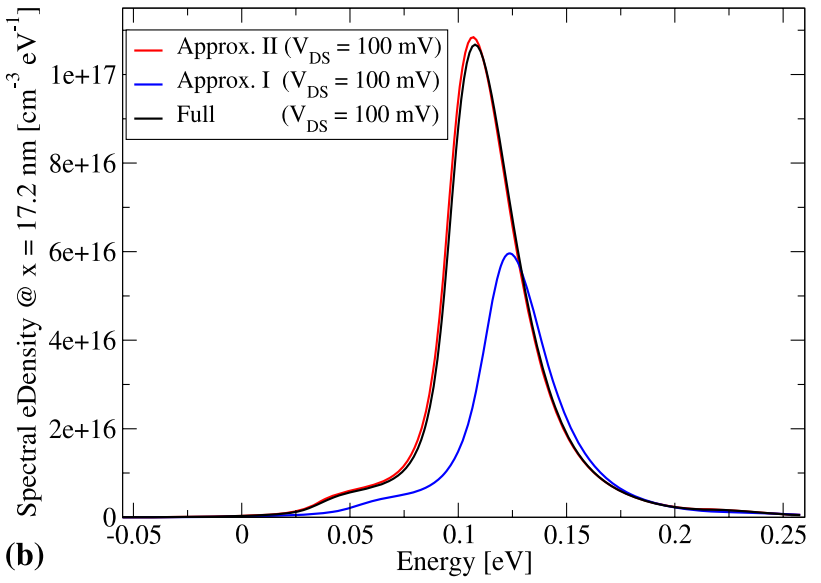

at $x=17.2 \mathrm{~nm}$ ) is shown in (b). Approximation $I$ leads to significant underestimation of the charge, while Approximation II is able to reproduce the charge of the full solution

tant once there are many electrons in the device with high energies, i.e. their location in the Brillouin zone deviates from the conduction band edge, where the EMA approach is valid. However, in a non-equilibrium system, scattering results in a redistribution of the electrons in energy and momentum. From (44) it can be seen, that phonon emission is the favored process for electrons compared to phonon absorption, provided that the final states at $E \pm \hbar \omega_{q}$ exist and are unoccupied. This means that electrons tend to dissipate energy in the drain extension by relaxing to the conduction band edge, which alleviates the importance of nonparabolic band structure effects. If the initial energy of the electron is very high (compared to the conduction band edge and the respective chemical potential), as it is the case for an elec- 


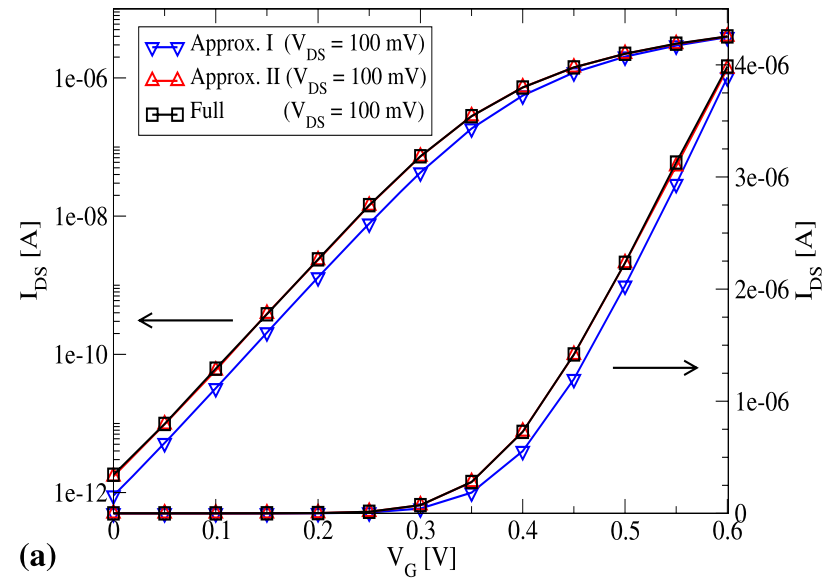

Fig. $7 \quad I_{\mathrm{DS}} V_{\mathrm{G}}$ curves for the different approximations of the retarded self-energy compared to the full solution. The error of the current using Approximation II is less than $1 \%$ over the whole voltage range, while

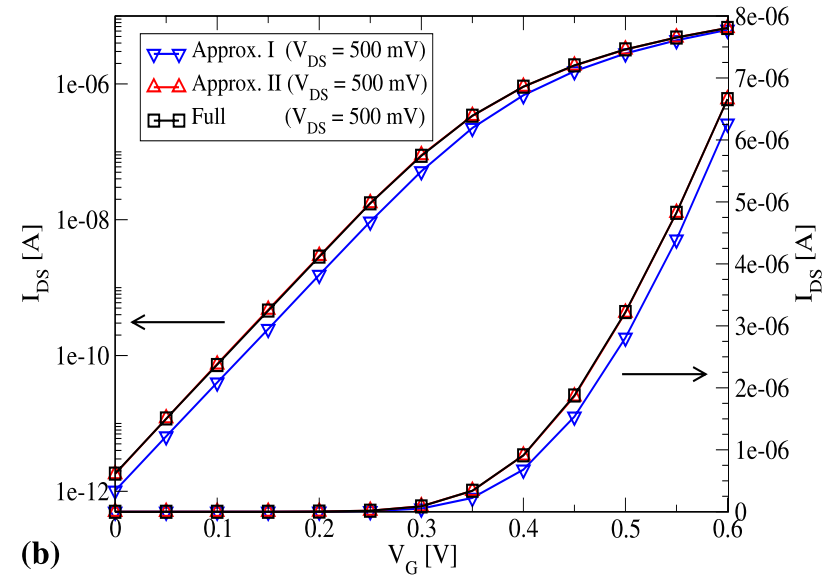

the subtreshold current of Approximation I yields an underestimation up to $80 \%$
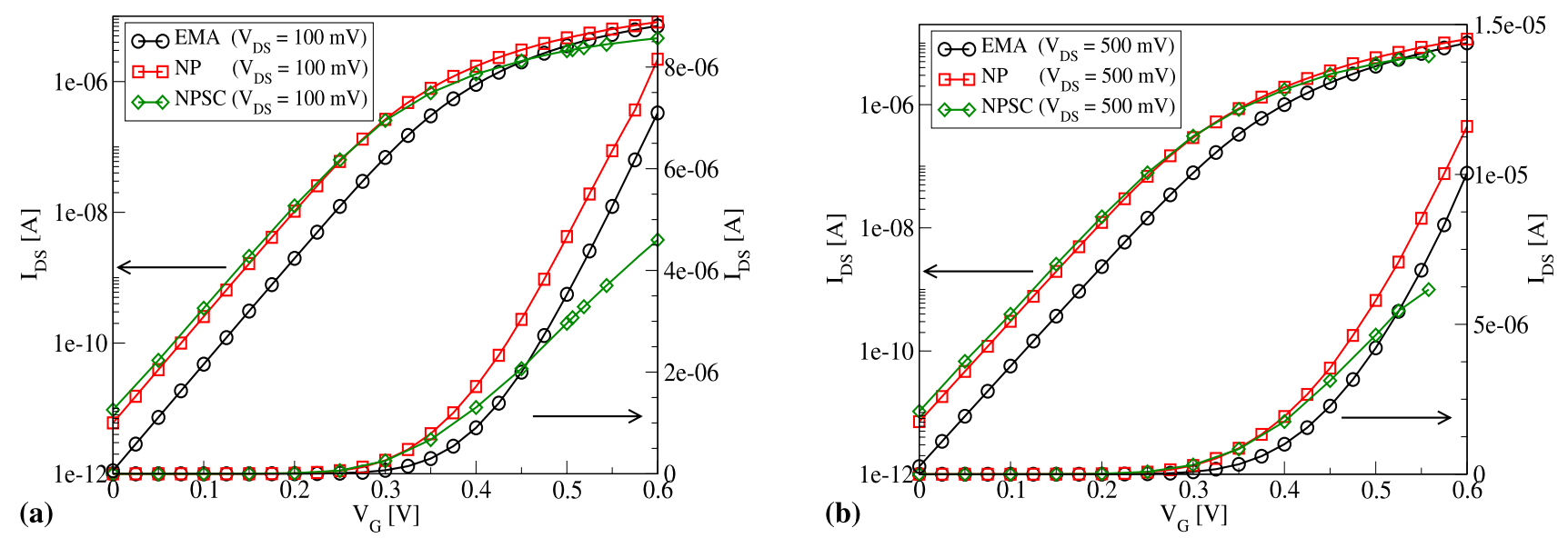

Fig. 8 Comparison of $I_{\mathrm{DS}} V_{\mathrm{G}}$ curves resulting from nonparabolicity (NP) and nonparabolicity with scattering (NPSC), using Approximation II. As a reference, the ballistic EMA curve is shown. Results are summarized in Table 3
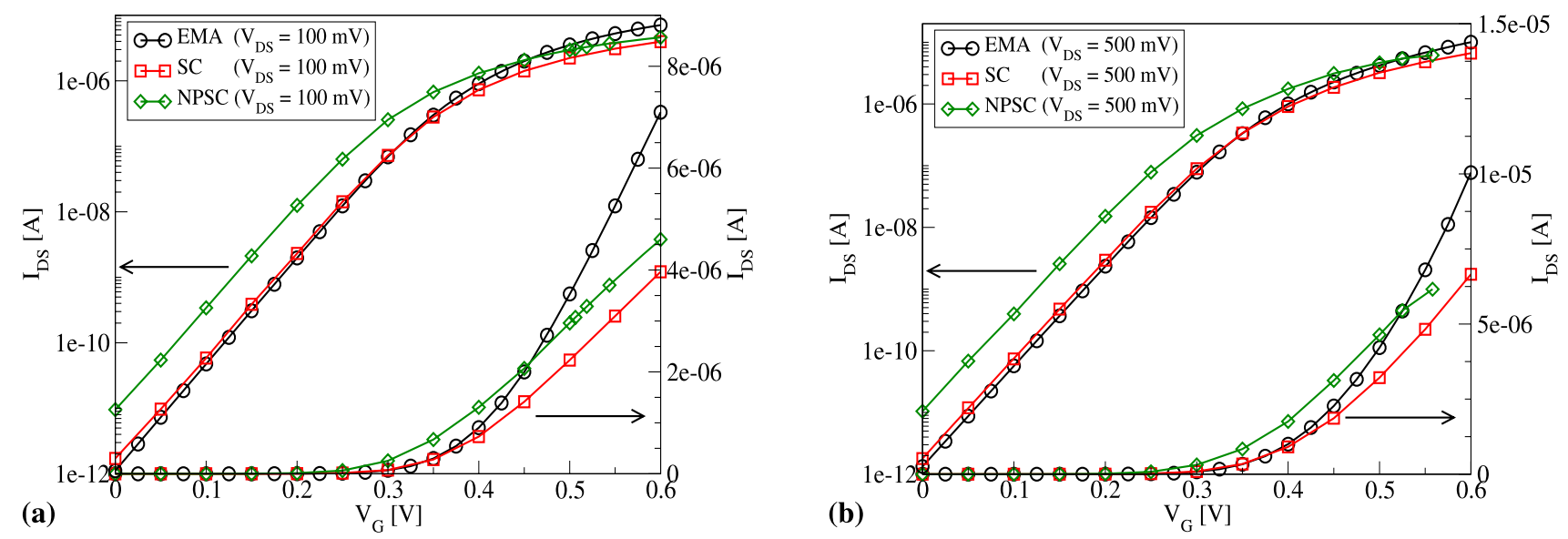

Fig. 9 Comparison of $I_{\mathrm{DS}} V_{\mathrm{G}}$ curves resulting from scattering (SC) and nonparabolicity with scattering (NPSC), using Approximation II. As a reference, the ballistic EMA curve is shown. Results are summarized in Table 3 
Table 3 Threshold voltages and ON-currents related to the transfer characteristics plotted in Figs. 8 and 9. Shown are the results of the two ballistic simulations, EMA and nonparabolicity $\mathrm{NP}$, as well as two simulations with scattering using Approximation II, SC and in combination with nonparabolicity NPSC. Two source-to-drain biases have been considered: $V_{\mathrm{DS}}=0.1 \mathrm{~V}(\mathrm{I})$ and $V_{\mathrm{DS}}=0.5 \mathrm{~V}(\mathrm{II})$. A spline interpolation is used to compute the data according to the definitions given in (48) and (49). The relative(REL) deviations $\left|I_{\mathrm{ON}}^{\text {ballistic }}-I_{\mathrm{ON}}^{\mathrm{NP}, \mathrm{SC}, \mathrm{NPSC}}\right| / I_{\mathrm{ON}}^{\text {ballistic }}$ and $\mid V_{\mathrm{T}}^{\text {ballistic }}-$ $V_{\mathrm{T}}^{\mathrm{NP}, \mathrm{SC}, \mathrm{NPSC}} \mid / V_{\mathrm{T}}^{\text {ballistic }}$ are shown as well

\begin{tabular}{lllll}
\hline mode & $V_{\mathrm{T}}(\mathrm{I})$ & $I_{\mathrm{ON}}(\mathrm{I})$ & $V_{\mathrm{T}}(\mathrm{II})$ & $I_{\mathrm{ON}}(\mathrm{II})$ \\
\hline EMA & $0.312 \mathrm{~V}$ & $7.53 \mathrm{e}-06 \mathrm{~A}$ & $0.308 \mathrm{~V}$ & $1.06 \mathrm{e}-05 \mathrm{~A}$ \\
NP & $0.266 \mathrm{~V}$ & $6.86 \mathrm{e}-06 \mathrm{~A}$ & $0.262 \mathrm{~V}$ & $9.25 \mathrm{e}-06 \mathrm{~A}$ \\
NP REL & $14.74 \%$ & $8.994 \%$ & $14.94 \%$ & $12.87 \%$ \\
SC & $0.311 \mathrm{~V}$ & $4.15 \mathrm{e}-06 \mathrm{~A}$ & $0.304 \mathrm{~V}$ & $6.81 \mathrm{e}-06 \mathrm{~A}$ \\
SC REL & $0.32 \%$ & $45.28 \%$ & $1.30 \%$ & $35.86 \%$ \\
NPSC & $0.265 \mathrm{~V}$ & $4.04 \mathrm{e}-06 \mathrm{~A}$ & $0.258 \mathrm{~V}$ & $6.16 \mathrm{e}-06 \mathrm{~A}$ \\
NPSC REL & $15.06 \%$ & $46.39 \%$ & $16.23 \%$ & $41.94 \%$ \\
\hline
\end{tabular}

tron surmounting the source-to-drain barrier in the subtreshold regime, it cannot be completely thermalized by inelastic scattering in the short drain extension of about $10 \mathrm{~nm}$. This results in a significant difference in the subtreshold regime between the two $I_{\mathrm{DS}} V_{\mathrm{G}}$ curves labeled SC and NPSC in Fig. 9. With decreasing barrier height, the current is carried by electrons with lower energy and the difference between the curves is reduced, i.e. the importance of NP decreases. This observation is consistent with the conclusions, that can be drawn from comparing the results of the two different source-to-drain biases $V_{\mathrm{DS}}=0.1 \mathrm{~V}$ and $V_{\mathrm{DS}}=0.5 \mathrm{~V}$ in Table 3 . In the case of $V_{\mathrm{DS}}=0.5 \mathrm{~V}$, electrons from the source arriving at the drain extension have a higher kinetic energy compared to those with $V_{\mathrm{DS}}=0.1 \mathrm{~V}$, and therefore the band structure effects in NPSC still have an impact, whereas for $V_{\mathrm{DS}}=0.1 \mathrm{~V}$, the value of $I_{\mathrm{ON}}$ seems to be completely determined by scattering.

\section{Conclusion}

The separate and combined effects of nonparabolicity and phonon scattering have been studied for triple-gate silicon nanowires. As far as approximations for the retarded selfenergy are concerned, only Approximation II is found to reproduce the results of the full problem, whereas Approximation I leads to a significant error in the current. The observed shift in the threshold voltage $V_{\mathrm{T}}$ was identified as a band structure effect. The ON-current $I_{\mathrm{ON}}$, on the other hand, is mainly determined by scattering. The remaining influence of nonparabolicity on $I_{\mathrm{ON}}$ strongly depends on the kinetic energy of the carriers in the drain extension, which is a function of the source-to-drain voltage $V_{\mathrm{DS}}$. The higher $V_{\mathrm{DS}}$, the stronger is the impact of nonparabolicity on $I_{\mathrm{ON}}$.
Acknowledgements The authors would like to thank Dr. M. Luisier for providing the TB data. This work was financially supported by the EU project IST-216171-NANOSIL.

\section{Appendix}

In a non-equilibrium system, the introduction of scattering results in a redistribution of carriers in their energy and momentum. An important constraint is that the current must be conserved, which will be demonstrated in this appendix. To keep the equations as short as possible, subband and space indices will be dropped. The expression for current conservation can be written as:

$$
\begin{aligned}
\nabla \cdot I= & \int \frac{d E}{2 \pi} \operatorname{Tr}\left(H G^{<}(E)-G^{<}(E) H\right) \\
= & \int \frac{d E}{2 \pi} \operatorname{Tr}\left(\Sigma^{R}(E) G^{<}(E)+\Sigma^{<}(E) G^{A}(E)\right. \\
& \left.-G^{R}(E) \Sigma^{<}(E)-G^{<}(E) \Sigma^{A}(E)\right) \\
= & \int \frac{d E}{2 \pi} \operatorname{Tr}\left(G^{<}(E)\left(\Sigma^{R}(E)-\Sigma^{A}(E)\right)\right. \\
& \left.+\left(G^{A}(E)-G^{R}(E)\right) \Sigma^{<}(E)\right) .
\end{aligned}
$$

For the last equality, the invariance of the trace of a matrix product has been used:

$\operatorname{Tr}(A B)=\operatorname{Tr}(B A), \quad A, B$ arbitrary matrices.

The decisive question now is, whether or not

$\left(\Sigma^{R}(E)-\Sigma^{A}(E)\right) \stackrel{?}{=}\left(\Sigma^{>}(E)-\Sigma^{<}(E)\right)$

is fulfilled for the approximations of the retarded selfenergy.

For Approximation I, the proof is trivial:

$$
\begin{aligned}
\left(\Sigma^{R}(E)-\Sigma^{A}(E)\right)= & \left(\frac{1}{2}\left(\Sigma^{>}(E)-\Sigma^{<}(E)\right)\right. \\
& \left.-\frac{1}{2}\left(\Sigma^{>}(E)-\Sigma^{<}(E)\right)^{\dagger}\right) \\
= & \left(\Sigma^{>}(E)-\Sigma^{<}(E)\right) .
\end{aligned}
$$

For Approximation II of an inelastic interaction, the proof is slightly more involved:

$$
\begin{aligned}
& \left(\Sigma^{R}(E)-\Sigma^{A}(E)\right) \\
& \quad=\int \frac{d \mathbf{q}}{(2 \pi)^{3}} e^{i \mathbf{q}\left(\mathbf{r}-\mathbf{r}^{\prime}\right)}\left|M_{q}\right|^{2}
\end{aligned}
$$




$$
\begin{aligned}
& \times\left(\left(N_{q}+1\right) G^{R}\left(E-\hbar \omega_{q}\right)+N_{q} G^{R}\left(E+\hbar \omega_{q}\right)\right. \\
+ & \frac{1}{2}\left(G^{<}\left(E-\hbar \omega_{q}\right)-G^{<}\left(E+\hbar \omega_{q}\right)\right) \\
& -\left(N_{q}+1\right) G^{A}\left(E-\hbar \omega_{q}\right)-N_{q} G^{A}\left(E+\hbar \omega_{q}\right) \\
& \left.+\frac{1}{2}\left(G^{<}\left(E-\hbar \omega_{q}\right)-G^{<}\left(E+\hbar \omega_{q}\right)\right)\right) \\
= & \int \frac{d \mathbf{q}}{(2 \pi)^{3}} e^{i \mathbf{q}\left(\mathbf{r}-\mathbf{r}^{\prime}\right)}\left|M_{q}\right|^{2} \\
& \times\left(\left(N_{q}+1\right)\left(G^{R}\left(E-\hbar \omega_{q}\right)-G^{A}\left(E-\hbar \omega_{q}\right)\right)\right. \\
& +N_{q}\left(G^{R}\left(E+\hbar \omega_{q}\right)-G^{A}\left(E+\hbar \omega_{q}\right)\right) \\
& \left.+\left(G^{<}\left(E-\hbar \omega_{q}\right)-G^{<}\left(E+\hbar \omega_{q}\right)\right)\right) \\
= & \int \frac{d \mathbf{q}}{(2 \pi)^{3}} e^{i \mathbf{q}\left(\mathbf{r}-\mathbf{r}^{\prime}\right)}\left|M_{q}\right|^{2} \\
& \times\left(\left(N_{q}+1\right)\left(G^{>}\left(E-\hbar \omega_{q}\right)-G^{<}\left(E-\hbar \omega_{q}\right)\right)\right. \\
& +N_{q}\left(G^{>}\left(E+\hbar \omega_{q}\right)-G^{<}\left(E+\hbar \omega_{q}\right)\right) \\
& \left.+\left(G^{<}\left(E-\hbar \omega_{q}\right)-G^{<}\left(E+\hbar \omega_{q}\right)\right)\right) \\
= & \left(\Sigma^{>}(E)-\Sigma^{<}(E)\right) .
\end{aligned}
$$

$$
\begin{aligned}
& -G^{>}(E)\left(N_{q} G^{<}\left(E-\hbar \omega_{q}\right)\right. \\
& \left.\left.+\left(N_{q}+1\right) G^{<}\left(E+\hbar \omega_{q}\right)\right)\right)
\end{aligned}
$$$$
=0 \text {. }
$$

For the last equality a change of variables must be performed: $E \rightarrow E+\hbar \omega_{q}$ for the first term in the third line of (63) and $E \rightarrow E-\hbar \omega_{q}$ for the second term in the third line of (63). Then the total integrand vanishes.

\section{References}

1. Nehari, K., Lannoo, M., Michelini, F., Cavassilas, N., Bescond, M., Autran, J.L.: Improved effective mass theory for silicon nanostructures. Appl. Phys. Lett. 93, 092103 (2008)

2. Esposito, A., Luisier, M., Frey, M., Schenk, A.: A nonparabolicity model compared to tight-binding: The case of square silicon quantum wires. Solid State Electron. 53, 376 (2008)

3. Rodríguez-Bolívar, S., Gómez-Campos, F.M., Carceller, J.E.: An atomistic-based correction of the effective-mass approach for investigating quantum dots. J. Appl. Phys. 104, 104309 (2008)

4. Gómez-Campos, F.M., Rodríguez-Bolívar, S., López-Villanueva, J.A., Jiménez-Tejeda, J.A., Carceller, J.E.: A solution of the effective-mass Schrödinger equation in general isotropic and nonparabolic bands for the study of two-dimensional carrier gases. J. Appl. Phys. 98, 033717 (2005)

5. Trellakis, A., Ravaioli, U.: Three-dimensional spectral solution of the Schrödinger equation for arbitrary band structures. J. Appl. Phys. 92, 3711 (2002)

6. López-Villanueva, J.A., Melchor, I., Cartujo, P., Carceller, J.E.: Modified Schrödinger equation including nonparabolicity for the study of a two-dimensional electron gas. Phys. Rev. B 48, 1626 (1993)

7. Godoy, A., Yang, Z., Ravaioli, U., Gámiz, F.: Effects of nonparabolic bands in quantum wires. J. Appl. Phys. 98, 013702 (2005)

8. Nehari, K., Cavassilas, N., Autran, J.L., Bescond, M., Munteanu, D., Lannoo, M.: Influence of band structure on electron ballistic transport in silicon nanowire MOSFET's: An atomistic study. Solid State Electron. 50, 716 (2006)

9. Wang, J., Rahman, A., Ghosh, A., Klimeck, G., Lundstrom, M.: On the validity of the parabolic effective-mass approximation for the I-V calculation of silicon nanowire transistors. IEEE TED 52, 1589 (2005)

10. Nehari, K., Cavassilas, N., Michelini, F., Bescond, M., Autran, J.L., Lannoo, M.: Full-band study of current across silicon nanowire transistors. Appl. Phys. Lett. 90, 132112 (2007)

11. Neophytou, N., Paul, A., Lundstrom, M.S., Klimeck, G.: Simulation of nanowire transistors: atomistic vs. effective mass models. J. Comput. Electron. 7, 363 (2008)

12. Gnani, E., Reggiani, S., Gnudi, A., Parruchini, P., Colle, R., Rudan, M., Baccarani, G.: Band-structure effects in ultrascaled silicon nanowires. IEEE TED 54, 2243 (2007)

13. Wang, J., Polizzi, E., Lundstrom, M.: A three-dimensional quantum transport simulation of silicon nanowire transistors with the effective-mass approximation. J. Appl. Phys. 96, 2192 (2004)

14. Kohn, W., Luttinger, J.M.: Motion of electrons and holes in perturbed periodic fields. Phys. Rev. 97, 869 (1954)

15. Luisier, M., Schenk, A., Fichtner, W., Klimeck, G.: Atomistic simulation of nanowires in the $s p^{3} d^{5} s^{*}$ tight-binding formalism. Phys. Rev. B 74, 205323 (2006) 
16. Heinz, F.O.: Simulation Approaches for Nanoscale Semiconductor Devices. Hartung Gorre, Konstanz (2004). http://e-collection. ethbib.ethz.ch/cgi-bin/show.pl?type=diss\&nr $=15435$

17. Albrecht, M., Song, B., Schnurpfeil, A.: A wave function based ab initio nonequilibrium Green's function approach to charge transport. J. Appl. Phys. 100, 013702 (2006)

18. Taylor, J., Guo, H., Wang, J.: Ab initio modeling of quantum transport properties of molecular electronic devices. Phys. Rev. B 63, 245407 (2001)

19. Heinz, F.O., Schenk, A.: Self-consistent modeling of longitudinal quantum effects in nanoscale double-gate metal oxide semiconductor field effect transistors. J. Appl. Phys. 100, 084314 (2006)

20. Keldysh, L.V.: Diagram technique for nonequilibrium processes. Sov. Phys. JETP 20, 1018 (1965)

21. Kadanoff, L.P., Baym, G.: Quantum Statistical Mechanics. Benjamin, New York (1965)

22. Venugopal, R., Ren, Z., Datta, S., Lundstrom, M.S, Jovanovic, D.: Simulating quantum transport in nanoscale transistors: Real versus mode-space approaches. J. Appl. Phys. 92, 3730 (2002)

23. Luisier, M., Schenk, A., Fichtner, W.: Quantum transport in twoand three-dimensional nanoscale transistors: Coupled mode effects in the nonequilibrium Green's function formalism. J. Appl. Phys. 100, 043713 (2006)
24. McLennan, M.J., Lee, Y., Datta, S.: Voltage drop in mesoscopic systems: A numerical study using a quantum kinetic equation. Phys. Rev. B 43, 13846 (1991)

25. Datta, S.: Electronic Transport in Mesoscopic Systems. Cambridge University Press, New York (2000)

26. Frey, M., Esposito, A., Schenk, A.: Boundary conditions for incoherent quantum transport. In: Proceedings of 13th IWCE 2009, p. 17 (2009)

27. Mahan, G.D.: Many-Particle Physics. Kluwer Academic/Plenum, New York (2000)

28. Jacoboni, C., Reggiani, L.: The Monte Carlo method for the solution of charge transport in semiconductors with applications to covalent materials. Rev. Mod. Phys. 55, 645 (1983)

29. Wacker, A.: Semiconductor superlattices: A model system for nonlinear transport. Phys. Rep. 357, 1 (2002)

30. Jin, S., Park, Y.J., Min, H.S.: A three-dimensional simulation of quantum transport in silicon nanowire transistor in the presence of electron-phonon interactions. J. Appl. Phys. 99, 123719 (2006)

31. Svizhenko, A., Anantram, M.P.: Role of scattering in nanotransistors. IEEE Trans. Electron. Devices 50, 1459 (2003)

32. Aeberhard, U., Morf, R.H.: Microscopic nonequilibrium theory of quantum well solar cells. Phys. Rev. B. 77, 125343 (2008) 